\title{
LHC searches for Dark Matter in compressed mass scenarios: challenges in the forward proton mode
}

\author{
L.A. Harland-Lang, ${ }^{a}$ V.A. Khoze, ${ }^{b, c}$ M.G. Ryskin ${ }^{c}$ and M. Tasevsky ${ }^{d}$ \\ ${ }^{a}$ Rudolf Peierls Centre, Beecroft Building, \\ Parks Road, Oxford, OX1 3PU, U.K. \\ ${ }^{b}$ IPPP, Department of Physics, University of Durham, \\ Durham, DH1 3LE, U.K. \\ ${ }^{c}$ Petersburg Nuclear Physics Institute, NRC "Kurchatov Institute", \\ Gatchina, St. Petersburg, 188300, Russia \\ ${ }^{d}$ Institute of Physics, Czech Academy of Sciences, \\ Na Slovance 2, 18221 Prague, Czech Republic \\ E-mail: lucian.harland-lang@physics.ox.ac.uk, V.A.Khoze@durham.ac.uk, \\ ryskin@thd.pnpi.spb.ru, Marek.Tasevsky@cern.ch
}

ABSTRACT: We analyze in detail the LHC prospects at the center-of-mass enery of $\sqrt{s}=$ $14 \mathrm{TeV}$ for charged electroweakino searches, decaying to leptons, in compressed supersymmetry scenarios, via exclusive photon-initiated pair production. This provides a potentially increased sensitivity in comparison to inclusive channels, where the background is often overwhelming. We pay particular attention to the challenges that such searches would face in the hostile high pile-up environment of the LHC, giving close consideration to the backgrounds that will be present. The signal we focus on is the exclusive production of same-flavour muon and electron pairs, with missing energy in the final state, and with two outgoing intact protons registered by the dedicated forward proton detectors installed in association with ATLAS and CMS. We present results for slepton masses of 120-300 GeV and slepton-neutralino mass splitting of $10-20 \mathrm{GeV}$, and find that the relevant backgrounds can be controlled to the level of the expected signal yields. The most significant such backgrounds are due to semi-exclusive lepton pair production at lower masses, with a proton produced in the initial proton dissociation system registering in the forward detectors, and from the coincidence of forward protons produced in pile-up events with an inclusive central event that mimics the signal. We also outline a range of potential methods to further suppress these backgrounds as well as to enlarge the signal yields.

KEYWORDS: Supersymmetry Phenomenology, QCD Phenomenology

ArXiv EPrint: 1812.04886 


\section{Contents}

1 Introduction 1

2 Signal cross section and selection cuts 3

3 Photon-initiated backgrounds 5

$3.1 \gamma \gamma \rightarrow W^{+} W^{-} \rightarrow l^{+} \nu+l^{-} \bar{\nu}$

3.2 Low mass $\gamma \gamma \rightarrow l^{+} l^{-}$production 8

4 QCD induced exclusive pair production $\quad 9$

5 Non-diffractive events with pile-up $\quad 10$

$\begin{array}{lll}5.1 & \text { FPD cuts } & 11\end{array}$

$\begin{array}{ll}5.2 \text { No charged cuts } & 13\end{array}$

$\begin{array}{lll}5.3 & \text { Di-lepton cuts } & 14\end{array}$

$\begin{array}{ll}\text { 5.4 Comparisons at zero pile-up } & 15\end{array}$

$\begin{array}{ll}\text { 5.5 Inclusive non-diffractive background at zero pile-up } & 15\end{array}$

$\begin{array}{lll}6 & \text { Results } & 15\end{array}$

$\begin{array}{lll}7 & \text { Conclusions } & 18\end{array}$

A Coherent, incoherent and evolution contributions to photon flux $\quad 19$

$\begin{array}{ll}\text { B Probability for proton hit via dissociation } & 20\end{array}$

\section{Introduction}

One of the main goals of the physics program at the LHC and future colliders is the search for beyond the Standard Model (BSM) physics. One well-motivated, and much explored, candidate among the existing BSM scenarios is the Minimal Supersymmetric Standard Model (MSSM), see e.g. [1-4] for reviews. This in particular offers a natural candidate for cold Dark Matter (DM), the Lightest Supersymmetric Particle (LSP), which is the stable lightest neutralino, $\widetilde{\chi}_{1}^{0}$, see e.g. [5, 6].

A possibility that has received significant recent attention in the context of LHC and future collider searches is the electroweak pair production of $R$-parity conserving states in compressed mass scenarios of supersymmetry (SUSY). That is, where the mass difference between the heavier state (e.g. the chargino, $\widetilde{\chi}^{ \pm}$, or slepton, $\left.\tilde{l}(g)\right)$ and the LSP $\widetilde{\chi}_{1}^{0}$ is small, see for instance [7-19]. Such models are well motivated by cosmological observations and naturalness considerations [20-24] as well as $(g-2)_{\mu}$ phenomenology [25-28]. In particular, 
the small mass splitting between the DM state and its charged partner plays a crucial role in bringing the dark matter abundance down into agreement with the observed value through co-annihilation mechanism [29-31].

As charginos and sleptons only interact electroweakly, their direct production cross sections at the LHC are quite small and correspondingly the LHC discovery potential and current experimental bounds are substantially weaker in comparison to other SUSY states [32, 33]. Moreover, searches in the compressed mass region via standard inclusive channels are experimentally very challenging, in particular because the SM $W W$ background produces a very similar final state, and therefore contaminates any potential signal. A further difficulty is that the visible decay products have low momenta and therefore often do not pass detector acceptance thresholds. In order to trigger on such events, generally the presence of an additional jet or photon due to initial state radiation is required, providing the final-state particles with a boost in the transverse plane, and thus generating a large missing transverse momentum.

With these challenges in mind, the potential to search for these comparatively light charged SUSY particles via photon-initiated production in hadron collisions has been widely discussed over the past few decades [34-38]. One clear benefit of considering this channel is its model independence, in sharp contrast to many other reactions. That is, the production cross sections are directly predicted in terms of the electric charges of the relevant states. Now, the development of the forward proton detectors (FPD) at the LHC allows us to perform a wide program of such searches [39-47]. In particular, dedicated AFP $[49,50]$ and CT-PPS [51] FPDs have recently been installed in association with both ATLAS and CMS, respectively. The purpose of these near-beam detectors is to measure intact protons arising at small angles, giving access to a wide range of Central Exclusive Production (CEP) processes

$$
p p \rightarrow p+X+p
$$

where the plus sign indicates the presence of the Large Rapidity Gaps (LRG) between the produced state and outgoing protons. The experimental signature for the CEP of electroweakinos is then the presence of two very forward protons that are detected in the FPD and two leptons from the $\tilde{l}(g) \rightarrow l+\widetilde{\chi}^{0}$ decay whose production vertex is indistinguishable from the primary vertex measured in the central detector. The well-defined initial state and presence of the tagged outgoing protons provides a unique handle, completely absent in the inclusive case, that is able to greatly increase the discovery potential.

The FPDs consist of tracking and timing detectors which are inserted in Roman Pots. They are placed at roughly $220 \mathrm{~m}$ from the interaction point (IP) on both sides of the ATLAS and CMS detectors. Their acceptance in the fractional momentum loss of the intact protons, $\xi$, is approximately $0.02<\xi<0.15$ at the nominal accelerator and beam conditions, which corresponds to the unprecedented acceptance of 250 to $1900 \mathrm{GeV}$ in the invariant mass of the central system $X$ when both protons remain intact and are registered in the detectors. An important advantage of these detectors is their excellent $\xi$ resolution, which permits a very precise measurement of the mass of central system, at the percent level. In the case of slepton pair production this allows a precise measurement 
of the slepton pair and then, keeping the average di-lepton mass low, this can provide a reasonably accurate determination of the mass of the LSP DM candidate.

In this paper we study in detail the LHC prospects for searching for such exclusive slepton pair production in compressed mass scenarios at the center-of-mass energy of $\sqrt{s}$ $=14 \mathrm{TeV}$. Such a possibility was first discussed in [44] (see also [52-55]), and has more recently been considered in [56]. Here, we perform for the first time a systematic analysis of the various challenges and sources of backgrounds that such studies must deal with, a serious consideration of which is essential to assess the potential of these exclusive channels. In particular, as well as the irreducible exclusive $W W$ background, we also consider the reducible backgrounds from semi-exclusive lepton pair production, where a proton produced in the initial proton dissociation registers in the FPDs, and the pile-up background where two soft inelastic events coincide with an inelastic lepton pair production event. As we will see these two formally reducible backgrounds are expected to play a significant role during nominal LHC running conditions, and require close examination.

The outline of this paper is as follows. In section 2 we consider the signal and present the event selection procedure. In section 3 we discuss the photon-initiated backgrounds, due both to exclusive $W W$ production and semi-exclusive low mass lepton pair production. In section 4 we discuss the semi-exclusive QCD-initiated background due to low mass jet and meson pair production. In section 5 we discuss the background from inelastic events in combination with independent pile-up interactions. In section 6 we summarize our numerical results for the expected event yields. Finally, in section 7 we conclude, while in appendix A we discuss the treatment of inelastic photon interactions and in appendix $\mathrm{B}$ we describe how the probability of a proton hit in the FPD due to a proton dissociation system is calculated.

\section{Signal cross section and selection cuts}

As in [15] we confine ourselves to a simplified SUSY model and consider the direct pair production of smuons and selectrons $\tilde{l}_{L, R}(l=e, \mu)$ only, where the subscripts $L, R$ denote the left- and right-handed partner of the electron or muon. The four sleptons are assumed to be mass degenerate and to decay with a $100 \%$ branching ratio into the corresponding SM partner lepton and $\widetilde{\chi}_{1}^{0}$ neutralino. We consider slepton masses in the $120-300 \mathrm{GeV}$ region, in order to be consistent with existing inclusive mass bounds [15, 19, 57] but provide experimentally feasible cross sections. To be concrete, we take four slepton mass points, 120, 200, 250 and $300 \mathrm{GeV}$, with in each case a relatively low mass splitting of $\Delta M=$ $M_{\tilde{l}}-M_{\widetilde{\chi}_{1}^{0}}=10 \mathrm{GeV}$ and $20 \mathrm{GeV}$, corresponding to the compressed scenario discussed above.

The di-lepton system of interest is defined by requiring an electron-positron or muonantimuon pair, with $p_{T}>5 \mathrm{GeV}$ and $|\eta|<2.5$ (or $|\eta|<4.0$ ) be present, ensuring that reasonable reconstruction and trigger efficiencies may be expected. Here, the enlarged $\eta$ coverage case corresponds to the ATLAS/CMS upgraded tracker for the High-Luminosity (HL)-LHC [58, 59]. Here, and for all exclusive processes below, we use the SuperChIC 2.07 Monte Carlo (MC) generator [46, 48] (the results of the more recent version 3 [47] will be very similar since the version 3 is updated to include heavy ion collisions but the aspects that deal with photon-initiated production in $p p$ collisions are essentially identical 
for the purposes of our study). We find that for such exclusive processes the signal cross section is rather low, ranging from $0.03 \mathrm{fb}$ to $0.8 \mathrm{fb}$ for the considered mass region.

It is therefore essential to select these events during nominal LHC running, where the instantaneous luminosity and hence pile-up activity is relatively high. As we will see, the average number of pile-up events, $\mu$, will significantly affect the size of the background, and with this in mind we will consider three reference points, namely $\mu=0,10$ and 50 , with the $\mu=0$ point only serving to disentangle pile-up from non-pile-up effects. In each case we will consider a reference integrated luminosity of $300 \mathrm{fb}^{-1}$, which can be accumulated within a few years by ATLAS and CMS experiments under current running conditions. After the HL-LHC upgrade collecting this amount of data would occur in a matter of months, however here even higher values of $\mu$ will be present and the prospects for running with FPDs in this environment requires close consideration, currently ongoing [60]. It should also be noted that at high $\mu$ expected at HL-LHC, keeping the rates of L1 triggers based on low- $p_{T}$ leptons low (and hence getting low pre-scales) will be challenging. We assume that it will be possible to sufficiently reduce them by constructing a trigger based on double-tagged protons and on topological and other cuts which will be explained below.

At high $\mu$, the probability of no pile-up interactions is essentially zero and therefore we cannot select exclusive events by requiring rapidity gaps be present in the central detectors. Instead we will apply a $z$-vertex veto, which requires that no vertices and tracks be present within $\pm 1 \mathrm{~mm}$ of the primary vertex. This procedure has been shown to be efficient in rejecting inclusive and pile-up backgrounds, while maintaining reasonable signal selection efficiencies, in the ATLAS measurements of exclusive muon pair [61] and $W W$ [62] production, and the CMS + TOTEM measurement of exclusive muon pair production [63].

The cuts we apply can be divided into three classes, namely: cuts on the detected protons in the FPDs ('FPD cuts'); requiring no other additional charged particles in the central detector ('no-charged cuts'); and the selection applied to the lepton pair ('di-leptons cuts'). The FPD cuts are applied at generator level by simply requiring both protons to be in a region where a sufficiently high acceptance can be expected, namely $0.02<\xi<0.15$. The lepton cuts are applied at generator level too but detector inefficiencies are accounted for by applying reconstruction efficiencies for electrons and muons as functions of their $p_{T}$. We take overall efficiencies as found in the inclusive slepton search [15]. This considers a similar lepton $p_{T}$ range (in fact in [15] an even lower $p_{T}>4 \mathrm{GeV}$ cut is applied) and includes all physics effects, in contrast to specialized combined performance studies which usually split various effects and show efficiencies for higher $p_{T}$. Nevertheless the lepton efficiencies taken from these inclusive slepton searches may in fact be overly conservative when applied to leptons in exclusive processes, which are not generally accompanied by additional particles. With this in mind we have applied the muon reconstruction efficiencies, which are typically by 10-15\% higher than the electron ones, for both muons and electrons in our study. These are to a good approximation flat in $\eta$ and are not expected to vary strongly with the amount of pile-up.

On the other hand, the efficiency of the no-charged or $z$-vertex veto cuts will vary with $\mu$, and so here we make use of the Delphes framework [64], a software package providing a fast simulation of detector response that takes into account the effect of magnetic field, the granularity of the calorimeters and sub-detector resolutions. Delphes is also able to 


\begin{tabular}{|c|c|c|}
\hline \multirow{2}{*}{ Efficiency } & \multicolumn{2}{|c|}{$\langle\mu\rangle_{\mathrm{PU}}$} \\
\cline { 2 - 3 } & 10 & 50 \\
\hline$z$-veto & 0.843 & 0.481 \\
\hline vertex & 0.100 & 0.391 \\
\hline tracks & 0.057 & 0.128 \\
\hline
\end{tabular}

Table 1. The signal $z$-vertex veto efficiency for the mass combination $M_{\tilde{l}} / M_{\widetilde{\chi}_{1}^{0}}=200 \mathrm{GeV} / 180 \mathrm{GeV}$ and two values of $\mu$. The 'vertex' ('tracks') row shows the fractions of events where at least one vertex (where no vertex but at least one track) is found in the region $\pm 1 \mathrm{~mm}$ from the primary vertex.

overlay a specified number of pile-up events with an existing MC signal or background event sample. For this study, input cards with ATLAS simulation parameters are supplied, while the pile-up events are generated with PүтніA 8.2 [65] in minimum bias (MB) mode. Table 1 shows the $z$-vertex veto efficiency for one specific signal sample defined by $M_{\tilde{l}} / M_{\widetilde{\chi}_{1}^{0}}$ $=200 \mathrm{GeV} / 180 \mathrm{GeV}$, but similar values are observed for the other mass combinations. Here, and throughout this paper we will consider two pile-up scenarios, namely $\langle\mu\rangle=10,50$. While the latter is relevant for nominal LHC running, the former is taken to give an idea of the scaling of the various efficiencies and event numbers we consider with pile-up, although the precise value is not of direct experimental relevance. The efficiency is defined in a sample of events containing at least two leptons with $p_{T}>5 \mathrm{GeV}$ and $|\eta|<2.5$, as a ratio of events that have no additional vertices and tracks in the region of $\pm 1 \mathrm{~mm}$ around the primary vertex, to all selected signal events. The $z$-vertex veto efficiencies are found to be in agreement with those estimated in [61,62]. For events that do not pass the $z$-vertex veto requirements, the 'vertex' row in table 1 shows the fraction of those that had at least one vertex with $\left|z_{\mathrm{vtx}}-z_{\mathrm{prim}}\right|<1 \mathrm{~mm}$ and the 'tracks' row shows the fraction of those that had all vertices with $\left|z_{\mathrm{vtx}}-z_{\text {prim }}\right|>1 \mathrm{~mm}$, but there was at least one track with $\left|z_{\text {trk }}-z_{\text {prim }}\right|<$ $1 \mathrm{~mm}$. Note that all ratios in one column sum up to $100 \%$.

All applied cuts in this analysis are summarized in table 2. Some of these are chosen in order to suppress specific background contributions, and will be explained in the following sections. After applying all cuts specified in table 2 and applying the lepton efficiencies as described above, the resulting signal event yields for an integrated luminosity of $300 \mathrm{fb}^{-1}$ are given in table 3. For completeness, we include here the single dissociation (SD) and double dissociation (DD) contributions, where one or two proton from the dissociation system registers in the FPDs; this will be discussed more later. Here we note that while the SD contribution reaches $6-9 \%$ for a mass of $120 \mathrm{GeV}$, they are below $1.5 \%$ elsewhere and the DD contribution is completely negligible. Enlarging the pseudorapidity range to $|\eta|<4.0$ increases the signal yields by $10 \%$ at most.

\section{Photon-initiated backgrounds}

\section{$3.1 \quad \gamma \gamma \rightarrow W^{+} W^{-} \rightarrow l^{+} \nu+l^{-} \bar{\nu}$}

The production of a $W W$ pair followed by leptonic decays via the same photon-initiated production mechanism as the signal is one of the major sources of background. Here, the 


\begin{tabular}{|c|c|c|}
\hline \multirow{4}{*}{ Di-lepton } & $5<p_{T, l_{1}, l_{2}}<40 \mathrm{GeV}$ & $\left|\eta_{l_{1}, l_{2}}\right|<2.5(4.0)$ \\
\cline { 2 - 3 } & Aco $\equiv 1-\left|\Delta \phi_{l_{1} l_{2}}\right| / \pi>0.13(0.095)$ & $2<m_{l_{l} l_{2}}<40 \mathrm{GeV}$ \\
\cline { 2 - 3 } & $\Delta R\left(l_{1}, l_{2}\right)>0.3$ & $\left|\eta_{l_{1}}-\eta_{l_{2}}\right|<2.3$ \\
\cline { 2 - 3 } & $\bar{\eta} \equiv\left|\eta_{l_{1}}+\eta_{l_{2}}\right| / 2<1.0$ & ||$p_{T l_{1}}|-| p_{T_{2}}||>1.5 \mathrm{GeV}$ \\
\cline { 2 - 3 } & $W_{\text {miss }}>200 \mathrm{GeV}$ & \\
\hline FPD & $0.02<\xi_{1,2}<0.15$ & $p_{T, \text { proton }}<0.35 \mathrm{GeV}$ \\
\hline No-charge & No hadronic activity & z-veto \\
\hline
\end{tabular}

Table 2. Cuts used in this analysis.

\begin{tabular}{|c|c|c|c|c|}
\hline \multirow{2}{*}{ scenario } & \multicolumn{4}{|c|}{ lepton $p_{T}$ interval $[\mathrm{GeV}]$} \\
\cline { 2 - 5 }$M_{\tilde{l}} / M_{\widetilde{\chi}_{1}^{0}}$ & $5-15$ & $5-20$ & $5-30$ & $5-40$ \\
\hline $120 / 100$ & 0.4 & 0.9 & 2.2 & 2.8 \\
\hline $120 / 110$ & 1.2 & 2.4 & 3.7 & 3.9 \\
\hline $200 / 180$ & 0.2 & 0.8 & 1.9 & 2.2 \\
\hline $200 / 190$ & 1.4 & 1.9 & 2.3 & 2.3 \\
\hline $250 / 230$ & 0.1 & 0.4 & 1.1 & 1.2 \\
\hline $250 / 240$ & 0.8 & 1.1 & 1.2 & 1.2 \\
\hline $300 / 280$ & 0.1 & 0.2 & 0.6 & 0.7 \\
\hline $300 / 290$ & 0.4 & 0.6 & 0.6 & 0.6 \\
\hline
\end{tabular}

Table 3. Signal event yields for integrated luminosity of $300 \mathrm{fb}^{-1}$ for four mass assignments and four lepton $p_{T}$ intervals after applying all cuts specified in table 2. Yields correspond to the sum of $\mu^{+} \mu^{-}$and $e^{+} e^{-}$final states observed in $|\eta|<2.5$ and to the sum of left and right-handed sleptons. Lepton reconstruction efficiencies taken from [15] as well as SD and DD contributions are included.

production cross section via the combined $e^{+} e^{-}$and $\mu^{+} \mu^{-}$decay channels is about $1 \mathrm{fb}$ prior to any final-state cuts, and so is somewhat larger than the signal. However, here the lepton $p_{T}$ is peaked at $\sim M_{W} / 2$, in contrast to the signal, which prefers lower values. We therefore place a $2<m_{l_{l} l_{2}}<40 \mathrm{GeV}$ cut on the di-lepton invariant mass and a related kinematic cut of $p_{T, l_{1}, l_{2}}<40 \mathrm{GeV}$, significantly reducing this background. An additional cut on the missing mass $W_{\text {miss }}>200 \mathrm{GeV}$, constructed from the momenta of the protons in the FPDs and of the leptons in the central detector, reduces this background further. This in particular corresponds to the invariant mass of the neutrino (neutralino) pair in the exclusive $W W$ (SUSY) cases, and therefore for the signal we must have $W_{\text {miss }}>2 m_{\widetilde{\chi}_{1}^{0}}$, while the background is peaked at much lower values. Distributions of these two variables for the signal and the exclusive $W W$ background are compared in figure 1. The di-lepton mass is plotted for all events before applying any cuts and the $W_{\text {miss }}$ distribution is shown after applying cuts on the lepton $p_{T}$ and di-lepton mass. As expected, both demonstrate a good potential in suppressing this background.

The expected numbers of background events after applying the cuts from table 2 for leptons in the $|\eta|<2.5$ region are summarized in table 4 . Very similar numbers are obtained for $|\eta|<4$.0. Because of the large central system masses, which must be larger than the 

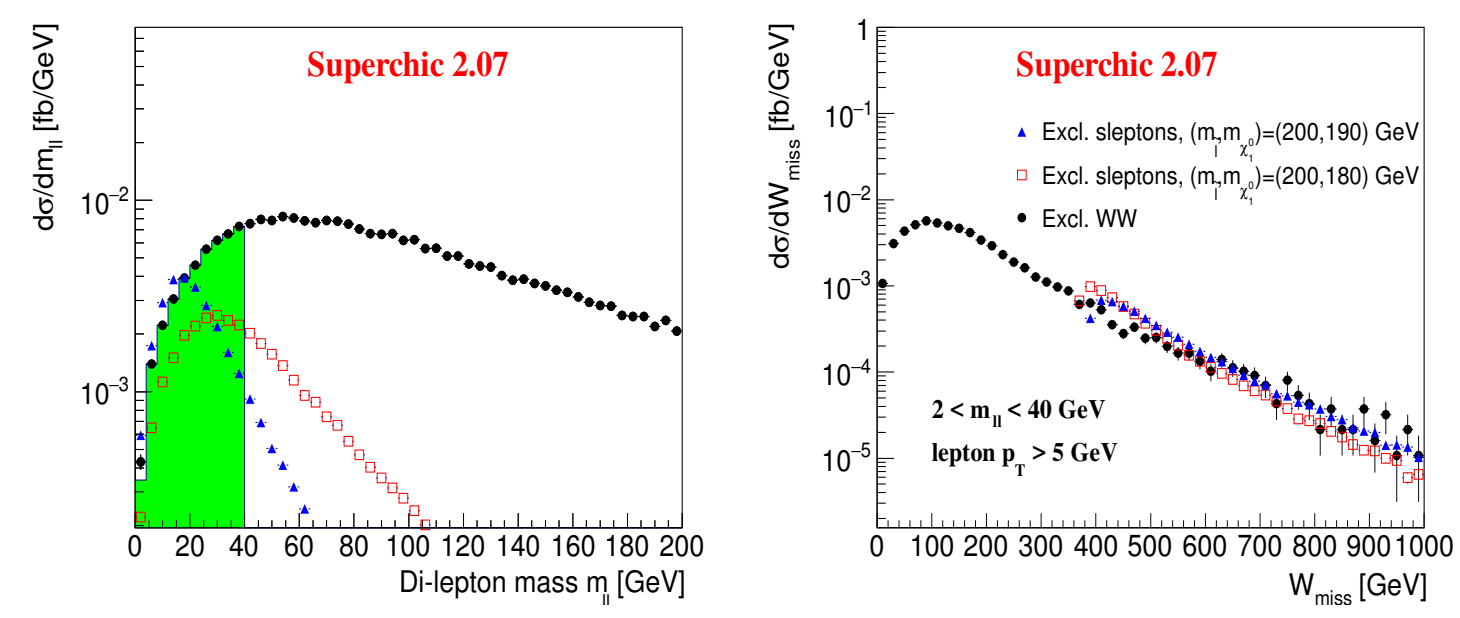

Figure 1. Distribution of di-lepton mass (left) and missing mass $W_{\text {miss }}$ (right) for exclusive slepton pair and $W W$ production. The di-lepton distribution was obtained before applying any cuts and the green area indicates the acceptance region used in the analysis. The $W_{\text {miss }}$ distribution was obtained after applying lepton $p_{T}$ and di-lepton mass cuts, see table 2.

\begin{tabular}{|c|c|c|c|c|}
\hline$W_{\text {miss }}>\mathrm{X}$ & \multicolumn{4}{|c|}{ lepton $p_{T}$ interval $[\mathrm{GeV}]$} \\
\cline { 2 - 5 }$[\mathrm{GeV}]$ & $5-15$ & $5-20$ & $5-30$ & $5-40$ \\
\hline $\mathrm{X}=200$ & 0.04 & 0.2 & 0.5 & 0.6 \\
\hline $\mathrm{X}=400$ & 0.03 & 0.1 & 0.3 & 0.3 \\
\hline $\mathrm{X}=500$ & 0.03 & 0.07 & 0.2 & 0.2 \\
\hline $\mathrm{X}=600$ & 0.02 & 0.05 & 0.1 & 0.1 \\
\hline
\end{tabular}

Table 4. Event yields at $300 \mathrm{fb}^{-1}$ for exclusive $W W$ production via leptonic decays, for four $W_{\text {miss }}$ cut values (corresponding to the four mass scenarios considered in this study) and four lepton $p_{T}$ intervals, after applying all cuts specified in table 2. Yields correspond to the sum of $\mu^{+} \mu^{-}$and $e^{+} e^{-}$final states observed in $|\eta|<2.5$. Lepton reconstruction efficiencies taken from [15].

$W_{\text {miss }}$ cut of $200 \mathrm{GeV}$, the SD and DD dissociation contributions (see the following section for definitions) are negligible, as the corresponding $\xi$ values fall out of the FPD acceptance. For our final results, we will consider the first row. The other rows would become relevant in the case of an observation, in which case by tuning the $W_{\text {miss }}$ cut threshold such that $W_{\text {miss }}>2 M_{\widetilde{\chi}_{1}^{0}}$, we would be able to suppress this background more strongly.

Finally, we note that the above situation is much worse for the case of chargino pair production. Though an obvious advantage of this process is the possibility to search in the final state for a pair of different flavour leptons (e.g. $e^{-}$and $\mu^{+}$), which allows to exclude the QED background from low mass lepton production (discussed below), here, the branching ratio for the leptonic decay is approximately 5 times smaller. After accounting for the larger (up to factor of 4) production cross section in the chargino case (see e.g. [38]), we find that the expected signal will be about 7-9 times smaller, and so the $W W$ background will strongly exceed the signal. 


\subsection{Low mass $\gamma \gamma \rightarrow l^{+} l^{-}$production}

The exclusive production of lepton pairs in the FPD acceptance region, i.e. with $m_{l_{l} l_{2}}>$ $280 \mathrm{GeV}$, has a very small production cross section and will moreover lead to extremely different final-state lepton kinematics in comparison to the signal. This will therefore not provide a source of background. On the other hand, the production cross section for lepton pairs in the $m_{l_{l} l_{2}}>10 \mathrm{GeV}$ region, corresponding to the $p_{T}>5 \mathrm{GeV}$ cuts in the central detector, is about $8.4 \mathrm{pb}$, that is many orders of magnitude higher than the signal. While purely exclusive production will in this case not produce protons in the FPD acceptance, the situation changes if we allow the outgoing protons to dissociate, that is consider semi-exclusive production. Here, a proton from the dissociation system may still be detected in the FPD, but with a $\xi$ value that no longer matches the rapidity and mass of the central system. While the probability that this occurs is very small, nonetheless in combination with the significantly larger di-lepton production cross section, the resulting background may be rather large. To calculate this probability, we can either apply an analytic expression for the leading proton distribution, as fit to soft hadronic data, or directly evaluate this using appropriate event samples generated with PYTHIA 8.2. As discussed further in appendix $\mathrm{B}$, these give very similar results. We in particular calculate this probability to be $P_{\text {SDnel }} \approx 0.7 \%$ (where the subscript indicates that this is due to protons from the inelastic side of a SD event) for a proton to lie in the FPD acceptance, which while small is in fact significantly larger than the suppression in the signal cross section relative to the $m_{l_{l} l_{2}}>10 \mathrm{GeV}$ di-lepton cross section. We therefore have to apply further cuts to suppress this source of background.

The cross sections for semi-exclusive lepton pair production were calculated by applying the procedure outlined in [66]. That is, we supplement the result of SuperCHIC 2.07 with 'effective' photon fluxes given according to elastic and low/high scale inelastic photon emission from the proton, but which pass the additional veto on central particle production due to the no-charged requirement. Both SD and DD contributions, where only one or both protons dissociate, respectively, are included. Further details are given in appendix A. We consider contributions from electron, muon and $\tau$ pair production, with the latter case followed by leptonic decays simulated by PYTHIA 8.2.

How can we reduce this background? First, we note that in the SD case, in order for a relatively low mass di-lepton to be produced with a sufficiently large $\xi$ value on the elastic proton side, the lepton pair must be produced at forward rapidity in the proton direction. Therefore, to suppress this contribution we require $\bar{\eta}=\left|\eta_{l_{1}}+\eta_{l_{2}}\right| / 2<1$. Furthermore, a $\left|\eta_{l_{1}}-\eta_{l_{2}}\right|<2.3$ cut rejects events with a large value of $m_{l_{l} l_{2}}$ but a rather small $p_{T l}$. However, after applying these cuts the low-mass $\gamma \gamma \rightarrow l^{+} l^{-}$background still exceeds the expected signal. To further reduce the background, due both to SD and DD, we introduce a cut on transverse momentum of the forward proton, $p_{T \text {,proton }}<0.35 \mathrm{GeV}$, as the $p_{T}$ of the proton produced in the dissociation system will generally be larger than that in the elastic case. This cut is applied directly in our calculation of appendix B, and reduces the probability for a proton from the dissociation system to be registered in the FPD significantly, to $P_{\text {SDnel }} \approx 0.4 \%$. In addition, in exclusive production the transverse momenta carried by the 


\begin{tabular}{|c|c|c|c|c|c|}
\hline \multirow{2}{*}{$\begin{array}{c}\text { Exclusive } \\
\text { di-lepton }\end{array}$} & Proton & \multicolumn{4}{|c|}{ lepton $p_{T}$ interval $[\mathrm{GeV}]$} \\
\cline { 3 - 6 } & dissociation & $5-15$ & $5-20$ & $5-30$ & $5-40$ \\
\hline$e^{+} e^{-}+\mu^{+} \mu^{-}$ & $\mathrm{SD}$ & $\sim 0 / \sim 0$ & $\sim 0 / \sim 0$ & $\sim 0 / \sim 0$ & $\sim 0 / \sim 0$ \\
\cline { 2 - 6 } & $\mathrm{DD}$ & $1.4 / 1.1$ & $1.4 / 1.1$ & $1.4 / 1.1$ & $1.4 / 1.1$ \\
\hline$\tau^{+} \tau^{-}$ & $\mathrm{SD}$ & $0.01 / 0.00$ & $0.03 / 0.02$ & $0.05 / 0.02$ & $0.05 / 0.02$ \\
\cline { 2 - 6 } & $\mathrm{DD}$ & $\sim 0 / \sim 0$ & $\sim 0 / \sim 0$ & $\sim 0 / \sim 0$ & $\sim 0 / \sim 0$ \\
\hline
\end{tabular}

Table 5. Event yields for an integrated luminosity of $300 \mathrm{fb}^{-1}$ for di-lepton production in four lepton $p_{T}$ intervals after applying all cuts specified in table 2 . Results for single and double proton dissociation are given, and with $|\eta|<2.5 /|\eta|<4.0$ intervals for the final-state lepton. Lepton reconstruction efficiencies are taken from [15]. The purely exclusive contribution is exactly zero due to the mass acceptance of the FPDs and cuts imposed on $m_{l_{l} l_{2}}$, so are not shown. The values marked as $\sim 0$ correspond to numbers which are sufficiently below 0.01 .

initial-state photons are generally very small and hence to good approximation the leptons are produced back-to-back, with equal and opposite transverse momenta, that is with close to zero acoplanarity. While allowing for proton dissociation will generally increase the average photon $q_{T}$, and hence wash this out somewhat, nonetheless the background is significantly more peaked at low acoplanarity in comparison to the signal, where the leptons from the slepton decay can be produced at arbitrary $\phi$. We therefore apply an additional acoplanarity cut, $1-\left|\Delta \phi_{l_{1} l_{2}}\right| / \pi>0.13(0.095)$, for leptons in the $|\eta|<2.5(|\eta|<4.0)$ range. We in addition apply a cut on the difference $\left|p_{T l_{1}}-p_{T l_{2}}\right|>1.5 \mathrm{GeV}$ (where $p_{T l_{i}}=\left|p_{T l_{i}}\right|$ ), which reduces the background for the same reason. The method for applying these cuts is explained in more detail in appendix A.

The result of imposing these cuts is summarized in table 5. For the SD case, we find that the lower $\xi$ threshold shifts the di-lepton mass spectrum to higher values and in combination with the $\bar{\eta}$ cut the onset of the di-lepton mass spectrum moves beyond the $40 \mathrm{GeV}$ threshold for electron/muon production. For $\tau$-pair production the di-lepton masses are on average lower but nevertheless we find that the SD background is in all cases under control, while in the DD case it is found to be negligible, see table 5. This is due to the impact of the branching ratio for the decay to a lighter lepton, and because the two neutrinos from a $\tau$ decay carry away a significant fraction of the $\tau$ energy, such that only $18 \%$ of leptons from the decay survive the lepton $p_{T}$ cut. On the other hand, we find a non-negligible contamination from electron and muon pair production via DD interactions.

\section{QCD induced exclusive pair production}

QCD-induced exclusive hadron production with leptonic decays may also mimic the signal final-state. We consider three classes of this background below, namely vector meson production, $K^{+} K^{-}$pair production and $D^{+} D^{-}$pair production.

In the case of vector meson ( $\rho, J / \psi$ etc) production with leptonic decays, these backgrounds can be straightforwardly removed by omitting the regions of lepton pair invariant mass corresponding to the meson masses. 
The CEP of $K^{+} K^{-}$pairs requires a more careful treatment, but we again find this to be negligible. In particular, the SupERCHIC 2.07 prediction for this process, with $m_{K K}>$ $10 \mathrm{GeV}$ and kaon $p_{T}>4 \mathrm{GeV}$ is $1.3 \mathrm{fb}$, that is of the order of the signal. However, as in the case of photon-initiated lepton pair production, protons from the purely exclusive process will not register in the FPDs, and so we must again consider SD and DD semi-exclusive production. Once we include the small probabilities for protons from the dissociation system to be registered, we find that this background is very small. This will be further reduced by the fact that not all kaons will decay in the central detector due to the nonnegligible kaon path length.

A potentially more significant source of background is due to the production of heavy mesons which have a reasonable branching to leptons plus neutrals, e.g. $D^{+} \rightarrow \mu^{+} \nu \pi^{0}$. While the CEP of $D^{+} D^{-}$pairs is not currently generated in SUPERCHIC 2.07, the CEP of $c \bar{c}$ pairs, a natural source of $D$-mesons, is implemented, and hence we use this to estimate the size of the corresponding background. The cross section for masses of the $c \bar{c}$ system $m_{c \bar{c}}>10 \mathrm{GeV}$ and quark $p_{T}>5 \mathrm{GeV}$ is found to be about $3 \mathrm{nb}$. In addition, we consider the production of a low mass $g g$ pair, which while having a very low probability to produce mesons which decay in such a way as to mimic the signal, nonetheless has a very large production cross section. We in particular find that this amounts to $\sim 2 \mu \mathrm{b}$ for the same mass and $p_{T}$ cuts applied to the parton-level cross section as in the $c \bar{c}$ case. We note that as the elastic and SD cross sections are measured to be roughly the same at HERA [67], we do not modify the gluon fluxes to account for dissociation and have simply used the cross sections given by SuperChic 2.07 for the elastic case. Again, for these low masses it is only SD and DD events followed by proton hits in the FPDs from the dissociation system that can pass the event selection, and this requirement will reduce the backgrounds considerably. Indeed, we find that after applying cuts from table 2, the expected number of events for luminosity of $300 \mathrm{fb}^{-1}$ is below 0.01 in all cases, and hence is under very good control.

\section{$5 \quad$ Non-diffractive events with pile-up}

Leptons with relatively low $p_{T}$ are produced copiously at LHC: the inclusive production cross section is about $10 \mathrm{nb}$ for $p_{T}>5 \mathrm{GeV}$ [68]. The main source of these is from semileptonic decays of heavy mesons (e.g. $D^{+} \rightarrow \mu^{+} \nu \pi^{0}$ ) and the decay of $W$-bosons, while the decay of kaons and pions inside the central detector will also contribute [69]. If such an inclusive event coincides with protons registered in the FPDs due to unrelated pile-up interactions, this can contribute as a considerable source of background. To evaluate the effect of this we generate the dominant source of inclusive lepton production, due to nondiffractive (ND) jet production, with both Pythia 8.2 [65] and Herwig 7.1 [70, 71]. We find that the cross sections for jet $p_{T}>7 \mathrm{GeV}$ (taken as a conservative lower value) are unsurprisingly very large, with HeRWIG 7.1 (PYThIA 8.2) giving 16 (27) mb. Note that here we have fully included initial-state radiation (ISR), final-state radiation (FSR) and multi-parton interactions (MPI) in order to completely model the background, including the underlying event (UE). 
From a practical point of view, this cross section is so much larger than the signal $(\sim 0.1 \mathrm{fb})$ that it is impossible to generate a sufficiently large initial background sample in order to accurately evaluate the impact of cuts that reduce the background to the signal level, that is by roughly 14 orders of magnitude. To bypass this issue, we have instead considered the three approximately factorized classes of cuts introduced in section 2 , see table 2. In particular, we consider these cuts to be sufficiently independent that the final rejection efficiencies can be taken as the product of those due to applying the cuts individually. We discuss the impact of these in turn below.

\subsection{FPD cuts}

We first evaluate the probability $P_{\mathrm{FPD}}$, defined by the ratio of 'double tag' (DT) events containing a proton passing the FPD cuts in table 2 on each side of the interaction point and passing the ToF criteria discussed below, to the total event number. As the probability of registering a proton in the FPDs increases with $\mu$, this will clearly be quite sensitive to the total amount of pile-up. Note that these protons are dominantly due to soft SD events, with the most significant source of background then being due to an overlay of three pile-up events, i.e. two soft SD events which each produce a proton on one side of the interaction point and a hard-scale ND event that produces two leptons which pass all other cuts. However, there is some contribution from soft ND events, which may also produce protons in the FPD acceptance, and therefore we use MB events (including both diffractive and non-diffractive contributions) to evaluate the total effect of pile-up interactions.

At high pile-up the probability of such MB events giving hits in the FPDs is significant, while the probability of the final-state in the central detector from the hard-scale ND event mimicking the signal is small but non-zero. It is therefore challenging to suppress this background to a tolerable level. However, an effective way to do this is to equip the FPDs with time-of-flight ( ToF) detectors. Then, by measuring precisely the arrival times of the two individual protons on each side, one is able to suppress the pile-up background significantly. In general for the signal the $z$-vertex coordinate calculated from the proton arrival time in the FPDs will coincide with the vertex position of the central event within the vertex resolution, whereas for the background these will not. The designed time resolution is $10 \mathrm{ps}[49,51]$ for both AFP and CT-PPS, and much progress has been made to achieve this already.

In the results below we will therefore include this rejection factor, calculated by requiring that the $z$ position of the central vertex coincides with that calculated from the ToF detectors within this $\sigma_{t}=10 \mathrm{ps}$ resolution. In more detail, we assume that the bunch longitudinal width is $7.5 \mathrm{~cm}$ for non-zero $\mu$, and we require that the difference between the two arrival times be within $2 \sigma_{t}$. Under these assumptions, we then generate ten million bunch crossings for each $\mu$ point, with a number of $\mathrm{MB}$ events in each case given by a Poisson distribution and with the $z$-coordinate of the primary vertex given by a Gaussian distribution. Considering all DT events within this sample, and calculating the corresponding difference in arrival times of the proton hits, we then calculate the fraction of events where the evaluated vertex lies within $\sigma_{t}$ (corresponding to $3 \mathrm{~mm}$ ) on either side of the 


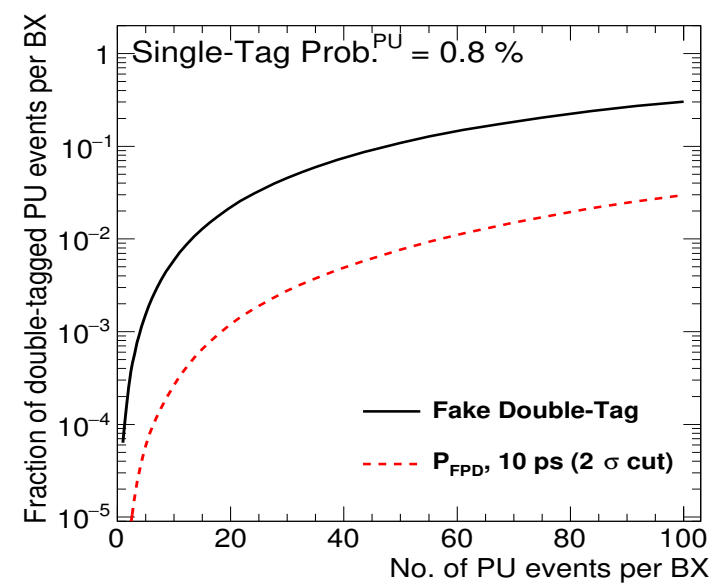

Figure 2. Fractions of fake DT events in one bunch crossing (BX) and overall rejection probabilities $P_{\mathrm{FPD}}$, including ToF discrimination, as a function of $\mu$. The results are based on a ST probability of 0.008 for MB events generated by PYTHIA 8.2 and on a ToF resolution of 10 ps.

\begin{tabular}{|c|c|c|c|c|}
\hline \multirow{2}{*}{} & \multicolumn{2}{|c|}{ PYTHIA 8.2 } & \multicolumn{2}{c|}{ HeRWIG 7.1 } \\
\cline { 2 - 5 } & \multicolumn{2}{|c|}{$\langle\mu\rangle_{\mathrm{PU}}$} & \multicolumn{2}{c|}{$\langle\mu\rangle_{\mathrm{PU}}$} \\
\cline { 2 - 5 } & 10 & 50 & 10 & 50 \\
\hline Fake DT & 0.0048 & 0.105 & 0.0123 & 0.222 \\
ToF rejection & 18.3 & 13.7 & 17.5 & 11.3 \\
$P_{\mathrm{FPD}}$ & $2.6 \times 10^{-4}$ & $7.6 \times 10^{-3}$ & $7.0 \times 10^{-4}$ & $2.0 \times 10^{-2}$ \\
\hline
\end{tabular}

Table 6. Probabilities of fake double-tagged events, rejection factors due to ToF detectors and overall probabilities to observe in one bunch crossing two forward protons in the FPDs and an inelastic vertex in the central detector consistent with that obtained by ToF detectors within the time resolution of $10 \mathrm{ps}$.

primary vertex. These results are summarized in figure 2. Note that the ToF reduction factors linearly depend on the inverse ToF resolution (for more details, see [72, 73]).

The calculated probabilities for a fake DT event to occur, and the ToF rejection factors are shown in table 6 . The total probability $P_{\mathrm{FPD}}$ is then given by the fake DT probability divided by the ToF rejection factor. We show results for both Pythia 8.2 and HerwiG 7.1 , and with two different values of $\mu$, and can see that the total rejection varies from 2 to 4 orders of magnitude, depending on the pile-up and generator used.

We can see that the results vary by a factor of $\sim 2$ depending on whether PYTHIA 8.2 or HeRWIG 7.1 is used. Considering the probability that a proton from an individual MB ('single-tag', ST) event passes the FPD cuts in table 2, we find a value of 0.008 (0.013) for Pythia 8.2 (Herwig 7.1). If we add the combinatoric effect of pile-up, for example for $\mu=50$, and use Poisson statistics, this probability increases to the value $\left(1-(1-0.008)^{49}\right) \approx 0.34$. The square of that, i.e. the fake DT probability, would then be 0.116 , in a good agreement with the exact value of 0.105 obtained by the procedure based on generating large samples of $\mathrm{MB}$ events described above. The overall probability $P_{\mathrm{FPD}}$ 
can then be approximately obtained by considering that the vertex must lie within a $6 \mathrm{~mm}$ window, in comparison to the total available region of $15 \mathrm{~cm}$, and then accounting for the combination of different arrival times between the two FPDs that may be consistent with the primary vertex gives, for $\mu=50$ and PYTHIA 8.2, an additional factor of 1.5. In total, the expected overall rejection factor is then $0.11 \times 0.6 \times 1.5 / 15 \sim 0.0066$, roughly consistent with the more precise results in table 6 .

More generally, the simulation of the inelastic pile-up events that lead to the DT hit are fundamentally due to soft QCD, and therefore there will clearly be some model dependence in these results. As a first check, we extract the ST probability, but with MPI turned off, and find that the impact is rather small increasing it by $\sim 10 \%$. On the other hand the $\xi$ spectra of the leading protons at LHC energies have not been tuned in the MC, as there is currently no LHC data available to do this. As a consistency check we therefore extract the ST (at $\mu=0$ ) probability at $\sqrt{s}=1.8 \mathrm{TeV}$, and find a value of $0.023(0.024)$ for Pythia 8.2 (Herwig 7.1), which are both consistent with CDF data [74] and with the prediction based on the triple-Regge vertex [75] developed to describe the ISR data, as well as the ABMST model prediction [76]. Thus at these lower energies where tuning has been performed for the $\xi$ spectra the two MCs agree with each and with theoretical expectations. We therefore conclude that the $\mathrm{ST}(\mu=0)$ probability is driven mainly by the overall energy scale, rather than the strength of MPI. In the future we may expect an improvement in the accuracy of the predictions at the LHC energies, as the models are refined and tuned to further LHC data.

\subsection{No charged cuts}

The probability to see no charged particles in the central detector other than two leptons for non-zero pile-up values is evaluated using the following relation:

$$
P_{\text {no-ch }}(\mu=10,50)=P_{\text {gap }}(\mu=0) \times P_{z-\text { veto }}(\mu=10,50)
$$

where $P_{\text {gap }}$ is estimated at particle level, while for $P_{z \text {-veto }}$ the values from table 1 are used. The latter was calculated using signal events generated by SuperCHIC 2.07, hadronized by PythiA 8.2 and with the ATLAS detector response simulated by Delphes. We note that the probability $P_{\text {gap }}(\mu=0)$ is not exactly zero in non-diffractive events (see for example [77]) as gaps may be generated by fluctuations in hadronization. Nonetheless, it is expected to be rather small. As the number of events with two leptons coming from background processes is very small, see below, we estimate $P_{\text {gap }}(\mu=0)$ using events selected so as to resemble the signal as closely as possible. In particular, we consider event samples with at least two charged particles with $p_{T}>5 \mathrm{GeV}$ and $|\eta|<2.5(4.0)$, where two of them are isolated by $\Delta R>0.3$, separately for each of the background processes with sufficiently high cross sections, namely $c \bar{c}$ and $g g$ CEP and inclusive ND jet production. We then evaluate $P_{\text {gap }}(\mu=0)$ as a fraction of events in this sample which have no additional charged particles with $p_{T}>0.4 \mathrm{GeV}$ and $|\eta|<2.5(4.0)$. Track reconstruction efficiencies in these $p_{T}$ and $\eta$ regions are satisfactory. The values of $P_{\text {no-ch }}$ for these processes and three values of pile-up are shown in table 7 . 


\begin{tabular}{|c|c|c|c|}
\hline \multirow{2}{*}{$P_{\mathrm{no}-\mathrm{ch}}$} & \multicolumn{3}{|c|}{$\langle\mu\rangle_{\mathrm{PU}}$} \\
\cline { 2 - 4 } & 0 & 10 & 50 \\
\hline CEP $c \bar{c}$ & $3.5 \times 10^{-3}$ & $2.9 \times 10^{-3}$ & $1.7 \times 10^{-3}$ \\
\hline CEP $g g$ & $3.3 \times 10^{-5}$ & $2.8 \times 10^{-5}$ & $1.6 \times 10^{-5}$ \\
\hline Incl. jets $(|\eta|<2.5)$ & $5.2(2.0) \times 10^{-7}$ & $4.4(1.7) \times 10^{-7}$ & $2.5(1.0) \times 10^{-7}$ \\
\hline Incl. jets $(|\eta|<4.0)$ & $1.7(0.7) \times 10^{-7}$ & $1.4(0.6) \times 10^{-7}$ & $0.8(0.3) \times 10^{-7}$ \\
\hline
\end{tabular}

Table 7. The no-charged rejection probabilities as a function of $\mu$ for $c \bar{c}$ and $g g$ CEP, and inclusive ND jet production. The numbers in the first column were obtained at particle level and then used to calculate the numbers in the other columns using eq. (5.1) and $P_{\mathrm{z}-\text { veto }}$ probabilities from table 1. The inclusive jet events were generated with Pythia 8.2 (HERWIG 7.1).

In principle, $P_{\text {no-ch }}$ can be obtained directly from the Delphes simulation by estimating

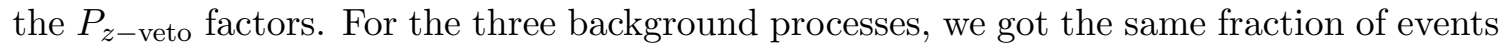
rejected by finding at least one pile-up vertex within $1 \mathrm{~mm}$ of the primary vertex as for the signal, namely $10 \%$ at $\mu=10$ and $39 \%$ at $\mu=50$ (see the 'vertex' row of table 1 ) and finally we obtained even lower values of $P_{z-\text { veto }}$ than $P_{\text {no-ch }}$. However, to be conservative we quote the higher values, as the primary vertex reconstruction algorithm implemented in Delphes requires further testing, in particular for the very high values of $\mu$ envisaged for the HL-LHC where it is not expected to distinguish all vertices and rather to merge some of them, especially in the region of beam spot (where the density of vertices is highest, see e.g. [78]).

In this context it is worth noting that if the $\eta$-coverage of the central tracker would be enlarged as approved for the ATLAS (so called ITk [58]) and CMS [59] upgrades for Run III, the probability $P_{\text {gap }}$ would decrease. Indeed, applying the same procedure as above and only requiring no charged particles in the range $p_{T}>0.4 \mathrm{GeV}$ and $|\eta|<4.0$, the probability $P_{\text {gap }}$ for inclusive events drops by a factor of three - as can be seen by comparing the inclusive jet rows in table 7 .

\subsection{Di-lepton cuts}

To evaluate the impact of the di-lepton cuts we select a sample of events where exactly two same-flavour and opposite sign leptons (electrons or muons) are produced with $p_{T}>5 \mathrm{GeV}$ and $|\eta|<2.5$ and satisfying most of the di-lepton cuts of table 2. Furthermore, we reject those events where the two selected leptons are accompanied by charged particles from the same heavy-meson decays. In other words, an event is rejected if at least one charged particle (besides the di-lepton pair) coming from the same decay as the lepton from the di-lepton pair has $p_{T}>0.4 \mathrm{GeV}$ and $|\eta|<2.5$. This effectively rejects decays modes with extra charged particles such as e.g. $D^{0} \rightarrow K^{-} e^{+} \nu$ or $D^{+} \rightarrow \rho^{0} \mu^{+} \nu$.

The probability $P_{\text {lep }}$ is then defined as the fraction of events surviving these cuts, and including the lepton reconstruction efficiencies from [15]. We find $P_{\text {lep }}=0.8 \times 10^{-7}(2.5 \times$ $10^{-7}$ ) with Pythia 8.2 (Herwig 7.1).* As mentioned in section 2, these reconstruction

\footnotetext{
${ }^{*}$ Note that, as Pythia 8.2 does not include $\mathrm{W}$ bosons in inclusive ND jets, we have estimated this contribution with HeRwig 7.1, see section 6.
} 
efficiencies are not expected to depend significantly on the amount of pile-up, so the $P_{\text {lep }}$ rejection factors were obtained at generator level only and with $\mu=0$, but can safely be used up to $\mu=50$.

\subsection{Comparisons at zero pile-up}

It is useful to compare and discuss the above results at zero pile-up, in order to further elucidate the differences between the two generators. The total jet cross sections, $P_{\mathrm{FPD}}$, $P_{\text {gap }}$ and $P_{\text {lep }}$ are all estimated as explained above using a sample of ND inclusive jet events with $p_{T}>7 \mathrm{GeV}$. The ratios of the Herwig 7.1 to Pythia 8.2 results are found to be $0.67,0.55,0.40$ and 3.3, respectively. We can therefore see that HERWIG 7.1 has a higher particle multiplicity in inclusive ND jet events in comparison to PYTHIA 8.2. In more detail we find that it produces roughly 4 times as many events with at least two charged hadrons with $p_{T}>5 \mathrm{GeV}$ and $|\eta|<2.5$ and about twice as many events with a di-lepton pair with $p_{T}>5 \mathrm{GeV}$ and $|\eta|<2.5$. For this reason, we find that $P_{\text {gap }}$ is almost by a factor of 2.5 times lower in Herwig 7.1 in comparison to Pythia 8.2.

Since PYthia 8.2 does not include the $W$ boson production in ND jets the value of $P_{\text {lep }}$ in PYTHIA is by a factor of 3.3 times lower. The $P_{\text {lep }}$ in PYTHIA is then corrected using the fraction of $W$ boson events in all di-lepton events found in HERWIG.

For our purposes, and in particular given the very small sizes of all background probabilities $P_{\mathrm{FPD}}, P_{\text {gap }}$ and $P_{\text {lep }}$, in some cases based on quantities which were not yet tuned at LHC (e.g. large rapidity gaps or $\xi$ spectrum of the leading proton), these differences are acceptable.

\subsection{Inclusive non-diffractive background at zero pile-up}

A final source of background we have to in general consider is due to events where the proton hits in the FPDs are produced by a single underlying inelastic interaction, which also produces a lepton pair in the central detector. Such a background would equally be present at $\mu=0$, as well as for realistic $\mu$ values. The probability, $P_{\mathrm{acc}}$, that this occurs is given by the product

$$
P_{\mathrm{acc}}=P_{\mathrm{FPD}}^{\prime} \cdot P_{\text {lep }} \cdot P_{\text {gap }},
$$

where $P_{\text {FPD }}^{\prime}$ was calculated using PYTHIA 8.2 including MPI, from the inclusive jet sample generated with $p_{T}>7 \mathrm{GeV}$, where at least two charged particles are observed with $p_{T}$ $>5 \mathrm{GeV}$ and $|\eta|<2.5$. We then calculate the fraction of events in which a proton in the FPD acceptance of table 2 on one side is observed. The total probability is given by squaring this. For a mixture of ND and SD events with a dynamically generated values of the soft survival probablity, $S^{2}$ (a quantity which is available from PyTHIA 8.2), we find $P_{\mathrm{FPD}}^{\prime}=(0.0004)^{2}$. Since the cross section for dijet production is $\sigma\left(p_{T}>7 \mathrm{GeV}\right)=27 \mathrm{mb}$ and $P_{\text {lep }}=0.8 \times 10^{-7}$ and $P_{\text {gap }}=5.2 \times 10^{-7}$, the expected background is negligible.

\section{$6 \quad$ Results}

We collect our results for the expected signal and background event yields in tables 8 and 9 . Here, the former case corresponds to $|\eta|<2.5$ (i.e. the current tracker coverage) while the 


\begin{tabular}{|l|c|c|c|}
\hline Event yields $/$ & \multicolumn{3}{|c|}{$\langle\mu\rangle_{\mathrm{PU}}$} \\
\cline { 2 - 4 } $\mathcal{L}=300 \mathrm{fb}^{-1}$ & 0 & 10 & 50 \\
\hline Excl. sleptons & $0.6-3.9$ & $0.5-3.3$ & $0.3-1.9$ \\
\hline Excl. $l^{+} l^{-}$ & 1.4 & 1.2 & 0.7 \\
\hline Excl. $K^{+} K^{-}$ & $\sim 0$ & $\sim 0$ & $\sim 0$ \\
\hline Excl. $W^{+} W^{-}$ & 0.7 & 0.6 & 0.3 \\
\hline Excl. $c \bar{c}$ & $\sim 0$ & $\sim 0$ & $\sim 0$ \\
\hline Excl. $g g$ & $\sim 0$ & $\sim 0$ & $\sim 0$ \\
\hline Incl. ND jets & $\sim 0(\sim 0)$ & $0.1(0.1)$ & $1.8(2.4)$ \\
\hline
\end{tabular}

Table 8. Final event yields corresponding to an integrated luminosity of $300 \mathrm{fb}^{-1}$ as a function of amount of pile-up events per bunch crossing for the slepton signal and all considered background processes. All numbers correspond to the di-lepton mass range $2<m_{l_{1} l_{2}}<40 \mathrm{GeV}$ and lepton 5 $<p_{T}<40 \mathrm{GeV}$ and a tracker coverage of $|\eta|<2.5$. The ranges in the signal event yields illustrate the spread obtained from the entire studied slepton mass range: the lower value comes from the $\left(M_{\tilde{l}}, M_{\tilde{\chi}_{1}^{0}}\right)=(300,280) \mathrm{GeV}$, the higher from the $\left(M_{\tilde{l}}, M_{\tilde{\chi}_{1}^{0}}\right)=(120,110) \mathrm{GeV}$ scenario. The value marked as $\sim 0$ corresponds to a number which is sufficiently below 0.01 . The inclusive ND jet events were generated with Pythia 8.2 (Herwig 7.1).

\begin{tabular}{|l|c|c|c|}
\hline Event yields $/$ & \multicolumn{3}{|c|}{$\langle\mu\rangle_{\mathrm{PU}}$} \\
\cline { 2 - 4 } $\mathcal{L}=300 \mathrm{fb}^{-1}$ & 0 & 10 & 50 \\
\hline Excl. sleptons & $0.7-4.3$ & $0.6-3.6$ & $0.3-2.1$ \\
\hline Excl. $l^{+} l^{-}$ & 1.1 & 0.9 & 0.5 \\
\hline Excl. $K^{+} K^{-}$ & $\sim 0$ & $\sim 0$ & $\sim 0$ \\
\hline Excl. $W^{+} W^{-}$ & 0.6 & 0.5 & 0.3 \\
\hline Excl. $c \bar{c}$ & $\sim 0$ & $\sim 0$ & $\sim 0$ \\
\hline Excl. $g g$ & $\sim 0$ & $\sim 0$ & $\sim 0$ \\
\hline Incl. ND jets & $\sim 0(\sim 0)$ & $0.03(0.05)$ & $0.6(0.7)$ \\
\hline
\end{tabular}

Table 9. The same as in table 8 but for the enlarged tracker coverage $|\eta|<4.0$.

latter corresponds to $|\eta|<4.0$ (i.e. the upgraded tracker coverage). To give a global picture, these results correspond to the full di-lepton mass range of $2<m_{l_{1} l_{2}}<40 \mathrm{GeV}$, although information about individual lepton $p_{T}$ ranges for processes where it is relevant can be found in tables 3, 4 and 5. In summary, we observe that in total 2-3 signal events for $300 \mathrm{fb}^{-1}$ can be expected, with a $S / B \sim 1$. We note that Pythia 8.2 and (HeRwig 7.1) give similar predictions for the contamination from the inclusive ND jets. These relatively small numbers therefore clearly do not correspond to a statistically significant observation. There are however various ways to improve this situation.

From the point of view of the phenomenological analysis presented here, the situation may be improved by cutting on the variable proposed in [38], namely the maximum kinematically allowed values of $m_{\tilde{\chi}}$ and $m_{\tilde{l}}$ assuming the signal decay chain. Following the approach of [38], we have checked that these cuts lead to some mild improvement in 
the signal significance over the background, though the interplay between these and other cuts on $W_{\text {miss }}$ and $m_{l_{1} l_{2}}, p_{T, l}$ is rather delicate and requires some fine-tuning. We may also expect some reduction in the low mass dilepton SD and DD backgrounds, but in the absence of a full MC implementation this cannot currently be calculated.

Experimentally, the signal yield can be doubled by taking all di-lepton masses into account. This would, however, not only increase the background but also the average dilepton mass itself and hence limit the possibility of estimating the unknown mass of the DM particle by measuring the central system mass via the FPDs. Another way to increase the signal yield would be to increase the lepton reconstruction efficiencies. In the ATLAS study [15] they start at $70 \%$ for muons and at $50 \%$ for electrons and slowly rise with increasing $p_{T}$, nevertheless since we deal with two leptons, any increase in the single-lepton efficiency could have a reasonable impact.

The background contamination, in turn, could be lowered by considering the following points. Since a big part of background leptons comes from decays of heavier particles, they originate from a vertex displaced from the primary one. It would thus be natural to consider restricting track longitudinal, $z_{0}$, and transverse, $d_{0}$, impact parameters to some small values for all found leptons in background samples (as is done for example when determining reconstruction efficiencies of primary vertices, see [78]). Actual LHC $b$-physics analyses rather use a pseudo-proper lifetime as a more appropriate observable to separate primary from secondary vertices. Furthermore, as discussed above, both ATLAS and CMS are upgrading their trackers to cover the additional region $2.4<|\eta|<4$. Both are also considering adding timing detectors in these forward areas with resolution of about $30 \mathrm{ps}[79,80]$. The time information for tracks in this forward area will improve the trackto-vertex association, leading to a performance similar to that in the central region for both jet and lepton reconstruction, as well as the tagging of heavy-flavour jets. The timing in the central detector will allow us to check that not only the longitudinal, $z$, coordinate but also the time of lepton pair emission, measured via the forward protons ToF detectors, coincides (or not in the PU case) with the values measured in the central detector. That is we acquire another ToF rejection factor in addition to that shown in table 6 .

Finally, the background from events with proton dissociation may in principle be suppressed by rejecting events with particles observed in the proton dissociation region, i.e. at large rapidity, with a good timing resolution potentially allowing these particles to be distinguished from those that originate from pile-up. Unfortunately, as it stands the detector coverage of ATLAS or CMS is not sufficient in this region to achieve this but building Zero Degree Calorimeters designed to be radiation hard and providing also timing information [81] is under discussion both in ATLAS and CMS.

Before concluding, it is worth comparing in more detail with the results of the alternative study presented in [56]. We note that here only those backgrounds due to genuinely exclusive production, dominantly $W^{+} W^{-}$pair production, are considered, while pile-up effects, ND, SD and DD production are not accounted for. Some care is therefore needed in making such a comparison. Considering the experimentally most promising scenario, $\left(M_{\tilde{l}}, M_{\widetilde{\chi}_{1}^{0}}\right)=(120,110) \mathrm{GeV}$, and taking for the sake of direct comparison the no pile-up scenario, we can see from table 8 that we expect 3.9 signal events and $0.7 W^{+} W^{-}$back- 
ground events, corresponding to a $\sim 1.5(2.6) \sigma$ significance for $100(300) \mathrm{fb}^{-1}$. From figure 3 of [56] we can see that a somewhat larger $\sim 2 \sigma$ significance at $100 \mathrm{fb}^{-1}$ is found. This is however entirely expected: our numbers presented even for the experimentally unrealistic no pile-up scenario include various cuts, as in table 2, that are imposed to reduce the SD and DD backgrounds and which will correspondingly reduce this signal significance. Indeed, removing these increases the signal significance such that it is indeed rather consistent with [56]. However, clearly this is not the experimentally relevant case, and we should instead take the high pile-up case in table 8 , and include the corresponding formally reducible SD, DD and ND jet backgrounds. In this case the significance is clearly lower, but nonetheless this presents a more realistic picture of the current situation.

\section{Conclusions}

In this paper we have discussed the prospects for searching for slepton pair production via leptonic decays in compressed mass scenarios at the LHC, via photon-initiated production. In this case the experimental signal is simple, comprising only four charged particles in the final state, namely two forward outgoing protons and two leptons in the central detector. Theoretically, such models are well motivated by cosmological and naturalness considerations as well as $(g-2)_{\mu}$ phenomenology. Moreover, in inclusive channels the production of electroweakinos in such compressed mass scenarios is highly challenging due to the low production cross sections and high backgrounds. The exclusive search channel therefore offers a potentially unique method to probe such challenging regions of SUSY parameter space. Furthermore, it has the advantage that the predicted production cross sections are model independent, being driven only by the electric charge of the produced states.

On the other hand, the predicted production cross sections are small, being $\sim 0.1$ $\mathrm{fb}$ or less after accounting for all relevant cuts and efficiencies, depending on the slepton mass in the experimentally allowed region. Therefore it is essential to collect these events at nominal LHC luminosities and for any backgrounds to be under very good control. In this work we have analysed in detail all major sources of background under these conditions, where in particular pile-up will be high. In more detail, we have considered: the irreducible photon-initiated $W W$ background; the reducible background from the semiexclusive photon-initiated production of lepton pairs and QCD-initiated production of gluon and $c$-quark jets (via leptonic decays of hadrons produced in hadronization) at low mass, where a proton produced in the initial proton dissociation registers in the forward proton detectors; the reducible pile-up background where (dominantly) two independent single-diffractive events coincide with an inelastic lepton pair production event. For the proton dissociation and pile-up backgrounds we have performed dedicated MC simulations, including most of relevant detector effects and efficiencies, in order to evaluate their impact as accurately as possible.

We have found that requiring that the lepton pair lie in the signal $m_{l_{1} l_{2}}<40 \mathrm{GeV}$ region, combined with further judicially chosen cuts on the lepton momenta leads to significant reductions in the background. The pile-up backgrounds are strongly reduced by the use of fast timing in the proton tagging detectors, which is certainly essential to perform 
such a measurement, as well as the aforementioned lepton cuts and a further cut on the proton transverse momentum. These also help to reduce the semi-exclusive backgrounds considerably. However, after accounting for all of these effects we find that the backgrounds from pile-up and semi-exclusive photon-initiated lepton pair production are nonetheless expected to be of the same order as the signal, with the irreducible $W W$ background being somewhat lower.

Nonetheless, the intention of our study is to be as comprehensive and to a certain extent as conservative as possible. We have therefore also discussed a variety of ways in which this situation could be improved upon, with the potential for increased tracker acceptance combined with timing detectors at the HL-LHC being particularly promising. While a detailed study of the possibilities at the HL-LHC is beyond the scope of the current work, this provides a strong motivation for further work on this area, and for collecting data with tagged protons there. Certainly the main backgrounds are in principle reducible and therefore with further investigation we may be able to reduce these further. The discussed search strategy could also be used to explore other simplified models for Dark Matter with small mass splitting between the DM and its charged co-annihilation partner.

\section{Acknowledgments}

We thank Torbjörn Sjöstrand, Kasuki Sakurai, David Cerdeño, Mike Albrow, Alexander Kupčo, Pavel Řezníček, Mike Hance, Zbyněk Drásal, Michal Marčišovský and Michael Murray for useful discussions. This work was supported by the Ministry of Education, Youth and Sports of the Czech Republic under the project LG15052. MGR thanks the IPPP at the University of Durham for hospitality. VAK acknowledges the support from the Royal Society of Edinburgh Auber award.

\section{A Coherent, incoherent and evolution contributions to photon flux}

In this appendix we discuss in more detail how the case of semi-exclusive photon-initiated production is accounted for. In particular the contribution to the photon flux from elastic emission $(p \rightarrow p+\gamma)$, incoherent emission $\left(p \rightarrow N^{*} / \Delta+\gamma\right)$ and DGLAP emission from quarks should be considered. While the incoherent is dominant for lower mass proton dissociation, the DGLAP contribution dominates at higher mass. All these contributions can be precisely accounted according to the procedure outlined in [82], however this corresponds to the purely inclusive case, whereas here we require that no particles be produced within the central detector acceptance. To achieve this, we apply the approach of [66], to calculate 'effective' photon fluxes which survive an additional veto on central particle production.

We are then interested in three classes of events, namely when both photons are emitted coherently ('elastic'), one is emitted coherently and the other incoherently ('single dissociation', SD) and both are emitted incoherently ('double dissociation', DD). In the default version of SuperChic 2.07 only the first, purely elastic case is generated. Thus while this case is always treated exactly, such that cuts on the final-state particles can be directly 
imposed, in the latter two cases an effective approach must be taken. In particular, photon fluxes due to incoherent and DGLAP emission are calculated as in [66] with a veto imposed on additional particle production in the relevant $\eta$ region, and with the acoplanarity cut imposed by limiting the $z, Q^{2}$ integral (see (13) of [66])

$$
q_{T}=\left(Q^{2}(1-z)\right)^{1 / 2}>\pi \cdot \mathrm{Aco} \cdot p_{T},
$$

which generates the photon flux. For simplicity, we take $p_{T} \sim \frac{m_{l_{1} l_{2}}}{2}$ for the lepton transverse momentum, but note that a more precise treatment could give a somewhat different value for the final flux.

For the case of the cut on the scalar difference $\left|p_{T l_{1}}-p_{T l_{2}}\right|$, the photon $q_{T}^{2}$ distribution was generated logarithmically between $q_{0}=0.5 \mathrm{GeV}$ and $m_{l_{1} l_{2}} / 2$, where the lower scale is chosen so as to include low mass incoherent emission. This is then translated into the lepton momenta by including the appropriate angular integration.

In both cases, these are then multiplied by average survival factors of 0.85 and 0.15 in the SD and DD cases, respectively.

\section{B Probability for proton hit via dissociation}

To calculate the probability that a proton produced in the original proton dissociation system registers in the FPDs, we have evaluated the fraction of SD events generated by PүтніA 8.2 where a proton on the dissociation side is found within $0.02<\xi<0.15$ region:

$$
P_{\mathrm{SD}, \mathrm{nel}} \simeq 0.007
$$

If in addition, the proton is required to have $p_{T}<0.35 \mathrm{GeV}$, it reduces to

$$
P_{\mathrm{SD}, \mathrm{nel}} \simeq 0.004
$$

Alternatively, if we consider the fraction of ND events, with MPI turned off, which from Feynman scaling arguments we may also expect to give some estimate of this probability, we find

$$
P_{\mathrm{ND}} \simeq 0.005
$$

We note that one can also apply the tools of Regge theory to get an analytic estimate of $P_{\mathrm{SD}, \text { nel }}$. In particular, we use the triple-Regge formula for the soft inclusive proton-proton cross section

$$
\frac{d \sigma}{d^{2} p_{T} d \xi}=\sum_{i, j} G_{i i j}\left(p_{T}^{2}\right)(1-\xi)^{1-2 \alpha_{i}\left(p_{T}^{2}\right)}
$$

where $G_{i i j}$ is the triple Reggeon vertex, and the sum is over all contributing Reggeons, with the requirement that $i \neq I P$, i.e. the Pomeron exchange contribution, which corresponds to the elastic case where the proton does not dissociate, is excluded.

We in particular apply the fit of [75], assuming that the leading proton distribution at the LHC can be described by the same triple-Regge parameterization as this fit, which 
was tuned to describe CERN-ISR data (note that the more recent fit of [76] gives rather similar results). The probability to observe the proton in the FPD is then simply given by

$$
P_{\mathrm{SD}, \mathrm{nel}}=\frac{1}{\sigma_{\text {inel }}} \int d \xi d^{2} p_{T} \frac{d \sigma}{d^{2} p_{T} d \xi} .
$$

where the integral is performed over the relevant $\xi$ and $p_{T}$ region. For $0.02<\xi<0.15$ we find

$$
P_{\mathrm{SD}, \mathrm{nel}} \simeq 0.01
$$

while imposing $p_{T}<0.35 \mathrm{GeV}$ in addition gives

$$
P_{\mathrm{SD}, \mathrm{nel}} \simeq 0.0046 \text {. }
$$

Thus this value of $P_{\mathrm{SD} \text {,nel }}$ is in remarkable agreement with the MC value $P_{\mathrm{SD} \text {,nel }}$, and the latter is used throughout.

Open Access. This article is distributed under the terms of the Creative Commons Attribution License (CC-BY 4.0), which permits any use, distribution and reproduction in any medium, provided the original author(s) and source are credited.

\section{References}

[1] H.E. Haber and G.L. Kane, The search for supersymmetry: probing physics beyond the standard model, Phys. Rept. 117 (1985) 75 [INSPIRE].

[2] S.P. Martin, A supersymmetry primer, hep-ph/9709356 [INSPIRE].

[3] MSSM Working Group collaboration, The minimal supersymmetric standard model: group summary report, talk given at the GDR (Groupement De Recherche) - Supersymetrie, April 15-17, Montpellier, France (1998), hep-ph/9901246 [INSPIRE].

[4] H. Baer and X. Tata, Weak scale supersymmetry: from superfields to scattering events, Cambridge University Press, Cambridge U.K. (2006).

[5] H. Goldberg, Constraint on the photino mass from cosmology, Phys. Rev. Lett. 50 (1983) 1419 [Erratum ibid. 103 (2009) 099905] [INSPIRE].

[6] J.R. Ellis et al., Supersymmetric relics from the Big Bang, Nucl. Phys. B 238 (1984) 453 [INSPIRE].

[7] T.J. LeCompte and S.P. Martin, Large Hadron Collider reach for supersymmetric models with compressed mass spectra, Phys. Rev. D 84 (2011) 015004 [arXiv:1105.4304] [INSPIRE].

[8] H. Baer et al., Physics at a Higgsino factory, JHEP 06 (2014) 172 [arXiv:1404.7510] [INSPIRE].

[9] A. Arbey et al., Physics at the $e^{+} e^{-}$linear collider, Eur. Phys. J. C 75 (2015) 371 [arXiv: 1504.01726] [INSPIRE].

[10] CMS collaboration, Search for dark matter and supersymmetry with a compressed mass spectrum in the vector boson fusion topology in proton-proton collisions at $\sqrt{s}=8 \mathrm{TeV}$, Phys. Rev. Lett. 118 (2017) 021802 [arXiv:1605.09305] [INSPIRE]. 
[11] CMS collaboration, Search for new physics in the compressed mass spectra scenario using events with two soft opposite-sign leptons and missing transverse momentum at $\sqrt{s}=13 \mathrm{TeV}$, CMS-PAS-SUS-16-025 (2016).

[12] T. Golling et al., Physics at a 100 TeV pp collider: beyond the Standard Model phenomena, CERN Yellow Report (2017) 441 [arXiv:1606.00947] [INSPIRE].

[13] ILD CONCEPT GROUP collaboration, Naturalness and light Higgsinos: why ILC is the right machine for SUSY discovery, PoS EPS-HEP2017 (2017) 306 [arXiv:1710.02406] [INSPIRE].

[14] V.V. Khoze, A.D. Plascencia and K. Sakurai, Simplified models of dark matter with a long-lived co-annihilation partner, JHEP 06 (2017) 041 [arXiv:1702.00750] [INSPIRE].

[15] ATLAS collaboration, Search for electroweak production of supersymmetric states in scenarios with compressed mass spectra at $\sqrt{s}=13$ TeV with the ATLAS detector, Phys. Rev. D 97 (2018) 052010 [arXiv:1712.08119] [INSPIRE].

[16] CMS collaboration, Searches for pair production of charginos and top squarks in final states with two oppositely charged leptons in proton-proton collisions at $\sqrt{s}=13$ TeV, JHEP 11 (2018) 079 [arXiv: 1807.07799] [INSPIRE].

[17] CMS collaboration, Search for electroweak production of charginos and neutralinos in multilepton final states in proton-proton collisions at $\sqrt{s}=13$ TeV, JHEP 03 (2018) 166 [arXiv: 1709.05406] [INSPIRE].

[18] CMS collaboration, Search for new physics in events with two soft oppositely charged leptons and missing transverse momentum in proton-proton collisions at $\sqrt{s}=13$ TeV, Phys. Lett. B 782 (2018) 440 [arXiv:1801.01846] [INSPIRE].

[19] E. Bagnaschi et al., Likelihood analysis of the pMSSM11 in Light of LHC 13 TeV data, Eur. Phys. J. C 78 (2018) 256 [arXiv:1710.11091] [INSPIRE].

[20] R. Barbieri and G.F. Giudice, Upper bounds on supersymmetric particle masses, Nucl. Phys. B 306 (1988) 63 [INSPIRE].

[21] B. de Carlos and J.A. Casas, One loop analysis of the electroweak breaking in supersymmetric models and the fine tuning problem, Phys. Lett. B 309 (1993) 320 [hep-ph/9303291] [INSPIRE].

[22] G.F. Giudice, T. Han, K. Wang and L.-T. Wang, Nearly degenerate gauginos and dark matter at the LHC, Phys. Rev. D 81 (2010) 115011 [arXiv:1004.4902] [InSPIRE].

[23] C. Han et al., Probing light higgsinos in natural SUSY from monojet signals at the LHC, JHEP 02 (2014) 049 [arXiv: 1310.4274] [INSPIRE].

[24] P. Schwaller and J. Zurita, Compressed electroweakino spectra at the LHC, JHEP 03 (2014) 060 [arXiv: 1312.7350] [INSPIRE].

[25] M.A. Ajaib et al., Neutralinos and sleptons at the LHC in light of muon $(g-2)_{\mu}$, Phys. Rev. D 92 (2015) 075033 [arXiv: 1505.05896] [INSPIRE].

[26] T. Ghosh, Neutralinos and sleptons at the LHC in Light of Muon Anomalous magnetic moment, Ph.D. thesis, Texas A-M, U.S.A. (2016).

[27] A. Kobakhidze, M. Talia and L. Wu, Probing the MSSM explanation of the muon g-2 anomaly in dark matter experiments and at a $100 \mathrm{TeV}$ pp collider, Phys. Rev. D 95 (2017) 055023 [arXiv: 1608.03641] [INSPIRE]. 
[28] M. Endo, K. Hamaguchi, S. Iwamoto and K. Yanagi, Probing minimal SUSY scenarios in the light of muon $g-2$ and dark matter, JHEP 06 (2017) 031 [arXiv:1704.05287] [INSPIRE].

[29] K. Griest and D. Seckel, Three exceptions in the calculation of relic abundances, Phys. Rev. D 43 (1991) 3191 [INSPIRE].

[30] M.J. Baker et al., The coannihilation codex, JHEP 12 (2015) 120 [arXiv:1510.03434] [INSPIRE].

[31] J. Edsjo and P. Gondolo, Neutralino relic density including coannihilations, Phys. Rev. D 56 (1997) 1879 [hep-ph/9704361] [INSPIRE].

[32] ATLAS collaboration, https://twiki.cern.ch/twiki/bin/view/AtlasPublic/SupersymmetryPublicResults.

[33] CMS collaboration, https://twiki.cern.ch/twiki/bin/view/CMSPublic/PhysicsResultsSUS.

[34] J. Ohnemus, T.F. Walsh and P.M. Zerwas, $\gamma \gamma$ production of nonstrongly interacting SUSY particles at hadron colliders, Phys. Lett. B 328 (1994) 369 [hep-ph/9402302] [INSPIRE].

[35] K. Piotrzkowski, Tagging two photon production at the CERN LHC, Phys. Rev. D 63 (2001) 071502 [hep-ex/0009065] [INSPIRE].

[36] N. Schul and K. Piotrzkowski, Detection of two-photon exclusive production of supersymmetric pairs at the LHC, Nucl. Phys. Proc. Suppl. 179-180 (2008) 289 [arXiv:0806.1097] [INSPIRE].

[37] V.A. Khoze, A.D. Martin, M.G. Ryskin and A.G. Shuvaev, A new window at the LHC: BSM signals using tagged protons, Eur. Phys. J. C 68 (2010) 125 [arXiv:1002.2857] [inSPIRE].

[38] L.A. Harland-Lang, C.H. Kom, K. Sakurai and W.J. Stirling, Measuring the masses of a pair of semi-invisibly decaying particles in central exclusive production with forward proton tagging, Eur. Phys. J. C 72 (2012) 1969 [arXiv:1110.4320] [INSPIRE].

[39] FP420 R\&D collaboration, The FP420 \&3 project: Higgs and new physics with forward protons at the LHC, 2009 JINST 4 T10001 [arXiv:0806.0302] [INSPIRE].

[40] LHC Forward Physics Working Group collaboration, LHC forward physics, J. Phys. G 43 (2016) 110201 [arXiv: 1611.05079] [INSPIRE].

[41] O. Kepka and C. Royon, Anomalous WW coupling in photon-induced processes using forward detectors at the LHC, Phys. Rev. D 78 (2008) 073005 [arXiv:0808.0322] [InSPIRE].

[42] C. Royon, Forward physics at the LHC: from the structure of the Pomeron to the search for $\gamma$-induced resonances, Acta Phys. Polon. B 47 (2016) 1781 [arXiv:1606.07675] [InSPIRE].

[43] L.A. Harland-Lang, V.A. Khoze and M.G. Ryskin, Photon-initiated processes at high mass, Phys. Rev. D 94 (2016) 074008 [arXiv: 1607.04635] [INSPIRE].

[44] V.A. Khoze, A.D. Martin and M.G. Ryskin, Can invisible objects be 'seen' via forward proton detectors at the LHC?, J. Phys. G 44 (2017) 055002 [arXiv: 1702.05023] [InSPIRE].

[45] C. Baldenegro, S. Fichet, G. von Gersdorff and C. Royon, Searching for axion-like particles with proton tagging at the LHC, JHEP 06 (2018) 131 [arXiv: 1803.10835] [INSPIRE].

[46] L.A. Harland-Lang, V.A. Khoze and M.G. Ryskin, Exclusive physics at the LHC with SuperChic 2, Eur. Phys. J. C 76 (2016) 9 [arXiv: 1508. 02718] [InSPIRE].

[47] L.A. Harland-Lang, V.A. Khoze and M.G. Ryskin, Exclusive LHC physics with heavy ions: SuperChic 3, Eur. Phys. J. C 79 (2019) 39 [arXiv:1810.06567] [InSPIRE]. 
[48] SuperCHIC code and documentation, http://projects.hepforge.org/superchic/ (2019).

[49] ATLAS collaboration, Technical design report for the ATLAS forward proton detector, CERN-LHCC-2015-009 (2015).

[50] ATLAS collaboration, Status of the AFP project in the ATLAS experiment, AIP Conf. Proc. 1654 (2015) 090001 [INSPIRE].

[51] M. Albrow et al., CMS-TOTEM precision proton spectrometer, CERN-LHCC-2014-021 (2014).

[52] V.A. Khoze, Photon-photon collisions at the LHC, invited talk at the International Conference Photon-2017, May 22-27, CERN, Geneva Switzerland (2017).

[53] V.A. Khoze, Challenges in searches for dark matter at the LHC in forward proton mode, invited talk at the Workshop on Diffraction and Low-x Physics, August 26-September 1, Reggio Calabria, Italy (2018).

[54] V. A. Khoze, Challenges in searches for dark matter at the LHC in forward proton mode, invited talk at the Workshop on $Q C D$ and Diffraction: Various Faces of $Q C D$, November 15-17, Cracow, Pooland (2018).

[55] M. Tasevsky, Future physics measurements with AFP, invited talk at the LHC Working Group on Forward Physics and Diffraction, December 7-8, Geneva, Switzerland (2017).

[56] L. Beresford and J. Liu, Photon collider search strategy for sleptons and dark matter at the $L H C$, arXiv: 1811.06465 [INSPIRE].

[57] CMS collaboration, Search for chargino pair production and top squark pair production in final states with two leptons in proton-proton collisions at $\sqrt{s}=13 \mathrm{TeV}$, CMS-PAS-SUS-17-010 (2017).

[58] ATLAS collaboration, Technical design report for the ATLAS inner tracker strip detector, CERN-LHCC-2017-005 (2017).

[59] CMS collaboration, The phase-2 upgrade of the CMS tracker, CERN-LHCC-2017-009 (2017).

[60] HL/HE-LHC physics workshop report, in preparation (2019).

[61] ATLAS collaboration, Measurement of the exclusive $\gamma \gamma \rightarrow \mu^{+} \mu^{-}$process in proton-proton collisions at $\sqrt{s}=13$ TeV with the ATLAS detector, Phys. Lett. B 777 (2018) 303 [arXiv: 1708.04053] [INSPIRE].

[62] ATLAS collaboration, Measurement of exclusive $\gamma \gamma \rightarrow W^{+} W^{-}$production and search for exclusive Higgs boson production in pp collisions at $\sqrt{s}=8$ TeV using the ATLAS detector, Phys. Rev. D 94 (2016) 032011 [arXiv:1607.03745] [INSPIRE].

[63] CMS, TOTEM collaboration, Observation of proton-tagged, central (semi)exclusive production of high-mass lepton pairs in pp collisions at $13 \mathrm{TeV}$ with the CMS-TOTEM precision proton spectrometer, JHEP 07 (2018) 153 [arXiv: 1803.04496] [INSPIRE].

[64] DELPHES 3 collaboration, DELPHES 3, a modular framework for fast simulation of a generic collider experiment, JHEP 02 (2014) 057 [arXiv: 1307.6346] [INSPIRE].

[65] T. Sjöstrand et al., An introduction to PYTHIA 8.2, Comput. Phys. Commun. 191 (2015) 159 [arXiv: 1410.3012] [INSPIRE].

[66] L.A. Harland-Lang, V.A. Khoze and M.G. Ryskin, The photon PDF in events with rapidity gaps, Eur. Phys. J. C 76 (2016) 255 [arXiv:1601.03772] [inSPIRE]. 
[67] V.A. Khoze, A.D. Martin and M.G. Ryskin, The extraction of the bare triple-Pomeron vertex: a crucial ingredient for diffraction, Phys. Lett. B 643 (2006) 93 [hep-ph/0609312] [INSPIRE].

[68] P. Bolzoni and G. Kramer, Inclusive lepton production from heavy-hadron decay in pp collisions at the LHC, Nucl. Phys. B 872 (2013) 253 [Erratum ibid. B 876 (2013) 334] [arXiv: 1212.4356] [INSPIRE].

[69] ATLAS collaboration, Dimuon composition in ATLAS at 7 TeV, ATLAS-CONF-2011-003 (2011).

[70] M. Bahr et al., HERWIG++ physics and manual, Eur. Phys. J. C 58 (2008) 639 [arXiv: 0803.0883] [INSPIRE].

[71] J. Bellm et al., HERWIG 7.0/HERWIG++ 3.0 release note, Eur. Phys. J. C 76 (2016) 196 [arXiv: 1512.01178] [INSPIRE].

[72] M. Tasevsky, Review of central exclusive production of the Higgs boson beyond the standard model, Int. J. Mod. Phys. A 29 (2014) 1446012 [arXiv:1407.8332] [InSPIRE].

[73] B.E. Cox, F.K. Loebinger and A.D. Pilkington, Detecting Higgs bosons in the $b \bar{b}$ decay channel using forward proton tagging at the LHC, JHEP 10 (2007) 090 [arXiv:0709.3035] [INSPIRE].

[74] K.A. Goulianos and J. Montanha, Factorization and scaling in hadronic diffraction, Phys. Rev. D 59 (1999) 114017 [hep-ph/9805496] [INSPIRE].

[75] A.B. Kaidalov et al., On determination of the triple pomeron coupling from the ISR data, Phys. Lett. B 45 (1973) 493.

[76] R.B. Appleby et al., The practical Pomeron for high energy proton collimation, Eur. Phys. J. C 76 (2016) 520 [arXiv: 1604.07327] [INSPIRE].

[77] V.A. Khoze et al., Diffraction and correlations at the LHC: definitions and observables, Eur. Phys. J. C 69 (2010) 85 [arXiv:1005.4839] [INSPIRE].

[78] ATLAS collaboration, Reconstruction of primary vertices at the ATLAS experiment in Run 1 proton-proton collisions at the LHC, Eur. Phys. J. C 77 (2017) 332 [arXiv:1611.10235] [INSPIRE].

[79] CMS collaboration, Technical proposal for a MIP timing detector in the CMS experiment phase 2 upgrade, CERN-LHCC-2017-027 (2017).

[80] ATLAS collaboration, A High-Granularity Timing Detector (HGTD) in ATLAS: performance at the HL-LHC, ATL-LARG-PROC-2018-003 (2018).

[81] M.J. Murray, private communication.

[82] A. Manohar, P. Nason, G.P. Salam and G. Zanderighi, How bright is the proton? A precise determination of the photon parton distribution function, Phys. Rev. Lett. 117 (2016) 242002 [arXiv: 1607.04266] [INSPIRE]. 